\title{
An Optimization Grey Bernoulli Model and Its Application in Forecasting Oil Consumption
}

\author{
Kai Xu $\mathbb{D},{ }^{1}$ Xinyu Pang, ${ }^{2}$ and Huiming Duan ${ }^{2}$ \\ ${ }^{1}$ School of International Business and Management, Sichuan International Studies University, Chongqing 400031, China \\ ${ }^{2}$ School of Science, Chongqing University of Posts and Telecommunications, Chongqing 400065, China \\ Correspondence should be addressed to Kai Xu; 99002142@sisu.edu.cn
}

Received 7 February 2021; Revised 19 April 2021; Accepted 13 May 2021; Published 2 June 2021

Academic Editor: Xin Ma

Copyright $(2021$ Kai Xu et al. This is an open access article distributed under the Creative Commons Attribution License, which permits unrestricted use, distribution, and reproduction in any medium, provided the original work is properly cited.

Energy consumption in the world is mainly dependent on fossil energy, and oil is one of the main energy sources. Accurate prediction of oil consumption can provide an important basis for national energy security, which can provide reference and early warning for the implementation of the environmental strategy developed by the government. According to the nonlinearity of the energy system, this paper uses the principle of the grey nonlinear prediction model $\operatorname{NGBM}(1,1)$ to improve the background value of the model, and by the simulated annealing algorithm, we put forward the optimized grey nonlinear model ONGBM $(1,1)$. At the same time, the model is applied to the oil consumption of China, Chile, Mexico, and Japan. Based on the validity analysis of the existing data of the four countries, the model $\operatorname{ONGBM}(1,1)$ is basically superior to the other six grey forecast models. Finally, $\operatorname{ONGBM}(1,1)$ is used to predict the oil consumption of the four countries in the next five years, which can provide effective information for energy economic policy.

\section{Introduction}

With the continuous development of the economy, the increasing consumption of fossil energy has caused high carbon emissions, which has led to a series of environmental and ecological problems. In particular, it is the main cause of global warming, greenhouse effect, and extreme weather. Ecologically sustainable development requires us to pay attention to energy consumption. Energy development is currently in a critical period of transformation and change, facing unprecedented opportunities and challenges. In terms of energy consumption results, nowadays, energy consumption in the world is mainly dependent on fossil energy. Oil is one of the major energy sources, and the proportion of oil in energy consumption shows a rising trend, and oil affects the development of the global economy. Thus, the prediction of oil consumption is a necessary basis for the formulation of economic and social plans and can also provide an important basis for national energy security.

In addition, with the rapid development of the world, the global economic structure has undergone major changes, and the energy structure has also changed accordingly. Therefore, some historical data may have little reference value for the current energy situation or the future energy development trend. Therefore, only the data of recent years are reliable for energy consumption forecasts. In a modelling system with small samples and little information, the grey prediction model has more advantage, which is suitable for some systems with unclear information, and its forecast is to generate the original data sequence, weaken the randomness of the original data, to mine the hidden laws in the data sequence, and finally simulate and predict the data through the reduction operation.

The grey model was proposed by Deng [1] based on the grey theory of small sample and poor information system. The grey prediction model is one of the core parts of the grey system, which is characterized by a few data modelling and simple modelling. At present, it is widely used in energy [2-5], transportation [6-9], environment [10,11], and other industries [12-18].

Grey prediction model is also expanded from $\operatorname{GM}(1,1)$ model for predicting exponential growth data to univariate 
discrete models DGM $(1,1)$ model and $\operatorname{NDGM}(1,1)$ model $[19,20]$, as well as the single variable nonlinear grey Verhulst model and grey Bernoulli NGBM $(1,1)$ model $[21,22]$. Since there are many influencing factors in the model, univariate model is extended to multivariate models, such as $\operatorname{GM}(1, \mathrm{~N})$, $\operatorname{NGM}(1, \mathrm{~N}), \operatorname{GMC}(1, \mathrm{~N})$, and other multivariate models [23-25]. At the same time, a lot of researchers work on improving the accuracy of the model, such as optimizing the background value of the grey model, using appropriate algorithms to optimize the parameters, and combining with other machine learning methods to improve the accuracy, which promotes the development and improvement of the grey theoretical system.

The grey prediction model has been widely used by domestic and foreign scholars when predicting energy consumption. Ma et al. [26] established a delayed fractionalorder grey prediction model to predict coal and natural gas consumption in Chongqing, China, using the grey Wolf optimization algorithm; Jia et al. [27] proposed a modified GM(1,1) model by Markov chain to predict coal consumption in Gansu Province; Wu et al. [28] used the new multivariable grey prediction model to forecast the power consumption in Shandong Province. Wang et al. [29] proposed the combinational optimization model of GM(1,1)-ARIMA based on the IOWGA operator to predict the total coal consumption in the United States. Duan et al. [30] proposed a new model based on the property of data accumulation and fractional-order accumulation and used particle swarm optimization algorithm to seek the optimal fraction and then predicted the crude oil consumption in China from 2015 to 2020 by the model. In addition, the authors in $[2-5,31-33]$ have effectively predicted various energy consumption methods, and the grey model has achieved good results in the field of energy.

The energy system presents obvious nonlinear characteristics in real life. Based on this characteristic, in this paper, the nonlinear grey Bernoulli NGBM $(1,1)$ prediction model was optimized, and its modelling principle was perfected by optimizing the background value of the model, the model nonlinearity is embodied, the model structure is optimized, and the model accuracy is improved.

The simulated annealing algorithm is combined with the probabilistic jumping to randomly find the global optimal solution of the objective function in the solution space. Compared with other greedy algorithms, which may be trapped in the local optimal solution and cannot find the solution, the simulated annealing algorithm can jump out of the local optimal solution probabilistically and eventually approach the global optimal solution. At present, many scholars of grey systems also apply this algorithm to optimize grey models. For example, Luo et al. [34] put forward a new grey CCRGM $(1,1)$ prediction model by using the simulated annealing algorithm to optimize and apply it to the prediction of clean energy; Pai and Hong [35] proposed a combined method based on support vector machines and the simulated annealing algorithms for annual electricity load forecasting. Guo et al. [36] optimized the grey prediction model based on the simulated annealing algorithm and applied it to landslide prediction. In this paper, the simulated annealing algorithm is used to calculate the optimal undetermined coefficient in consideration of the operation characteristics, time processing cost, and optimization effect of the algorithm. The model is applied to four countries, China, Chile, Mexico, and Japan, and the oil consumption data of the four countries are taken as the effectiveness analysis of the model. The optimized grey Bernoulli model NGBM $(1,1)$ is compared with the other six grey prediction models, which all show better results. So, the model is used to predict oil consumption in four countries over the next five years.

The remainder sections are organized as follows. Section 2 establishes the optimized grey Bernoulli $\operatorname{NGBM}(1,1)$. Section 3 analyzes the validity of the model by using the energy data of four countries. Section 4 forecasts the oil consumption of four countries over the next five years. Section 5 provides the conclusion.

\section{Grey Bernoulli Extension Model ONGBM $(1,1)$}

In this section, firstly, the grey Bernoulli model NGBM $(1,1)$ is summarized. According to the grey action of the model and the parameters of the model nonlinearity, the model is extended into a new grey Bernoulli extended model $\operatorname{ONGBM}(1,1, \mathrm{k}, \mathrm{c})$, and the properties of the model are studied.

2.1. Optimized Grey Bernoulli Model ONGBM $(1,1)$. The original sequence is

$$
X^{(0)}=\left(x^{(0)}(1), x^{(0)}(2), \ldots, x^{(0)}(n)\right) .
$$

The first-order accumulating generation sequence is defined as

$$
X^{(1)}=\left(x^{(1)}(1), x^{(1)}(2), \ldots, x^{(1)}(n)\right),
$$

where $x^{(1)}(k)=\sum_{i=1}^{k} x^{(0)}(i),(k=1,2, \ldots, n)$.

$Z^{(1)}$ is adjacent to the mean generation sequence of $X^{(1)}$ :

$$
Z^{(1)}=\left(z^{(1)}(2), z^{(1)}(3), \ldots, z^{(1)}(n)\right)
$$

where

$z^{(1)}(k)=1 / 2 x^{(1)}(k)+1 / 2 x^{(1)}(k-1),(k=2,3, \ldots, n)$.

Definition 1. The sequences $X^{(0)}, X^{(1)}$, and $Z^{(1)}$ are as shown in equations (1)-(3), so

$$
x^{(0)}(k)+a z^{(1)}(k)=b\left(z^{(1)}(k)\right)^{\gamma} .
$$

It is called the grey Bernoulli model and it is abbreviated as $\operatorname{NGBM}(1,1)$ model [23], and the whitening equation has the following forms:

$$
\frac{\mathrm{d} x^{(1)}(t)}{\mathrm{d} t}+a x^{(1)}(t)=b\left(x^{(1)}(t)\right)^{\gamma}
$$

where $\gamma$ is a real number.

The time response of the $\operatorname{NGBM}(1,1)$ model is given by 


$$
\hat{x}^{(1)}(k)=\left\{\left[\left(x^{(0)}(1)\right)^{1-\gamma}-\frac{b}{a}\right] e^{-\mathrm{a}(\mathrm{k}-1)(1-\gamma)}+\frac{b}{a}\right\}^{1 / 1-\gamma}, \quad k=2,3, \ldots, n .
$$

The reducing value of $x^{(0)}(k)$ is

$$
\hat{x}^{(0)}(k)=\hat{x}^{(1)}(k)-\hat{x}^{(1)}(k-1), \quad k=2,3, \ldots, n .
$$

The background value in Definition 1 is defined as follows [24]:

$$
z^{(1)}(k)=(1+\beta) x^{(1)}(k)-\beta x^{(1)}(k-1), \quad \beta \in(-1,+\infty) .
$$

So, the model is defined as follows.

Definition 2. The sequences $X^{(0)}, X^{(1)}$, and $Z^{(1)}$ are as shown in equations (1), (2), and (8), so

$$
x^{(0)}(k)+a z^{(1)}(k)=b\left(z^{(1)}(k)\right)^{\gamma} .
$$

It is the optimized model of the grey Bernoulli model and is called $\operatorname{ONGBM}(1,1)$ model for short. follows:

The parameters $a$ and $b$ of the $\operatorname{ONGBM}(1,1)$ model are as

$$
(a, b)^{T}=\left(B^{T} B\right)^{-1} B^{T} Y
$$

where the matrices $B$ and $Y$ are

$$
\begin{aligned}
& B=\left(\begin{array}{cl}
-z^{(1)}(2) & \left(z^{(1)}(2)\right)^{\gamma} \\
-z^{(1)}(3) & \left(z^{(1)}(3)\right)^{\gamma} \\
\vdots & \\
-z^{(1)}(n) & \left(z^{(1)}(n)\right)^{\gamma}
\end{array}\right), \\
& Y=\left(\begin{array}{c}
x^{(0)}(2) \\
x^{(0)}(3) \\
\vdots \\
x^{(0)}(n)
\end{array}\right) .
\end{aligned}
$$

The reduction function is given by equations (6) and (7).

2.2. Optimization of $\operatorname{ONGBM}(1,1)$ Model by the Simulated Annealing Algorithm. The idea of the simulated annealing algorithm (hereinafter referred to as SA) was first proposed by Metropolis et al. In 1983, Kirkpatrick et al. used it for combinatorial optimization and VLSI design. SA to solve combinatorial optimization problem is based on the similarity between solid material annealing process in physics and general combinatorial optimization problem. When a solid substance is annealed, it is usually warmed and melted first, so that the particles can move freely. Then, as the temperature drops, the particles also gradually form a lattice of low energy. If the temperature near the condensation point drops slowly enough, the solid must form the lowest energy ground state.
The specific steps of SA to solve the model are as follows.

Step 1. Initialization: for general data (not big fluctuations), set the variation range of $\beta$ as $(-1,1)$. An initial solution $\beta_{0}$ is selected at random within this range, and the corresponding target value $\varepsilon\left(\beta_{0}\right)$ is calculated. Set the initial temperature as $T_{0}$ and the ending temperature as $T_{f}$, generate a random number $\alpha \in(0,1)$ as the threshold, the cooling law: $T(t+1)=V . T(t), \quad V \in(0,1)$ is the annealing coefficient, and $t$ is the $t$ th iteration. Since $T$ must be reduced slowly, $V$ should be a number close to 1 . Finally, convergence conditions are set according to actual requirements, such as the magnitude of error, number of iterations, or ending temperature. If the data are large fluctuation data, the variation range of $\beta$ is set as $(-1,1)$.

Step 2. At temperature $T$, another point $\beta^{\prime}$ in the neighborhood of the current solution $\beta$ is selected as the new solution, and the difference between the objective function value $\varepsilon(\beta)$ and $\varepsilon\left(\beta^{\prime}\right)$ is calculated, $\triangle \varepsilon=\varepsilon(\beta)-\varepsilon\left(\beta^{\prime}\right)$.

Step 3. When $\triangle \varepsilon<0$, it means that the new solution $\beta^{\prime}$ is accepted; when $\triangle \varepsilon>0$, it means that the new solution $\beta^{\prime}$ is accepted according to probability $p=\exp (-(\triangle \mathcal{E} / T))$; and if $p>\alpha$, then the new solution $\beta^{\prime}$ is accepted, and $\beta=\beta^{\prime}$.

Step 4. Following the cooling rule: $T(t+1)=V . T(t)$, the temperature is lowered.

Step 5. Repeat Steps 2-4 until the convergence condition is satisfied.

\section{Verification of $\mathrm{ONGBM}(1,1)$ Model}

This section is mainly divided into three parts. The first part describes the evaluation indicators mainly adopted in this research. In order to measure the effectiveness of the model, two evaluation indicators APE and MAPE are quoted in the research process, and the calculation method is given in Section 3.1. In the second part, the model $\operatorname{ONGBM}(1,1)$ is applied to the data from different countries for verification, and the simulation results are compared and analyzed with the existing grey univariate models, including $\operatorname{NGBM}(1,1)$, Verhulst, $\operatorname{GM}(1,1), \operatorname{NGM}(1,1)$, $\operatorname{TDGM}(1,1)$, and ARGM $(1,1)$ models. Meanwhile, the APE trend comparison diagram and MAPE comparison diagram of different cases have been given in Section 3.2. In the third part, based on the cases in the second part, the annual oil consumption in different regions in the next five years is predicted and analyzed. In the following example analysis, the calculation 


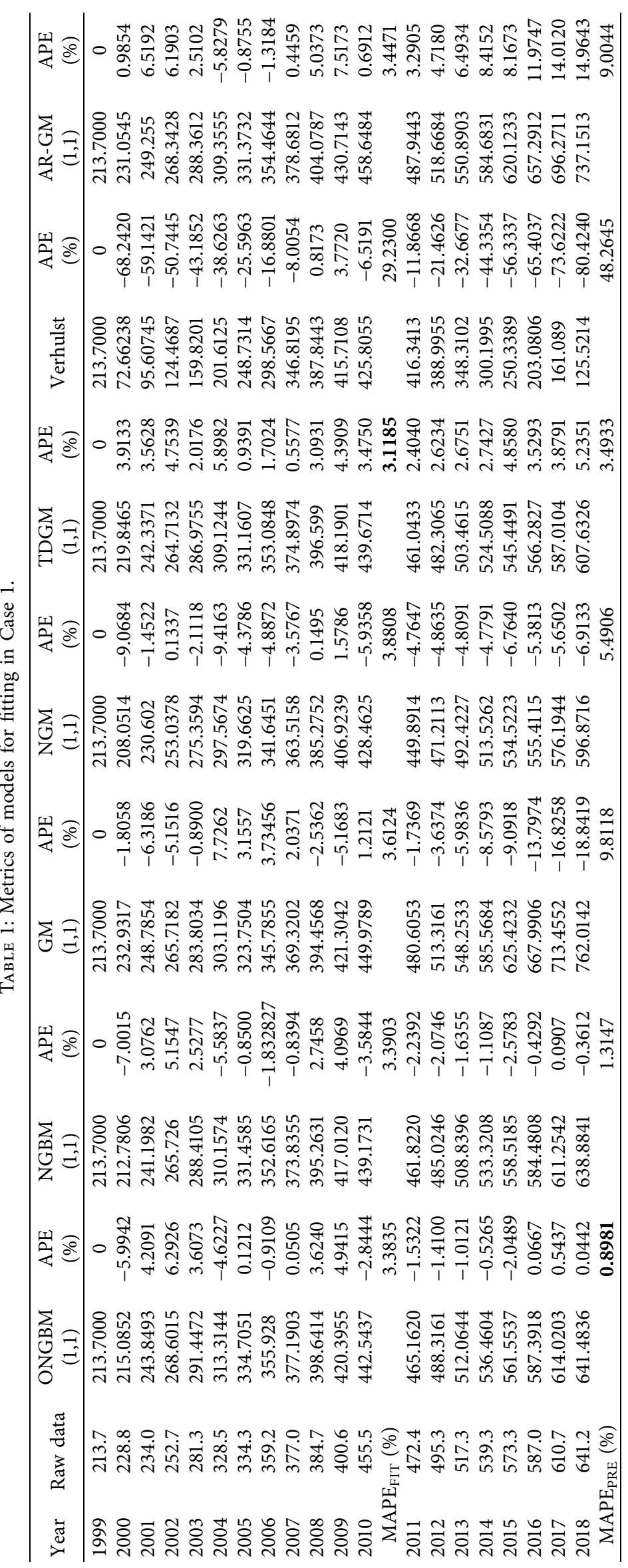




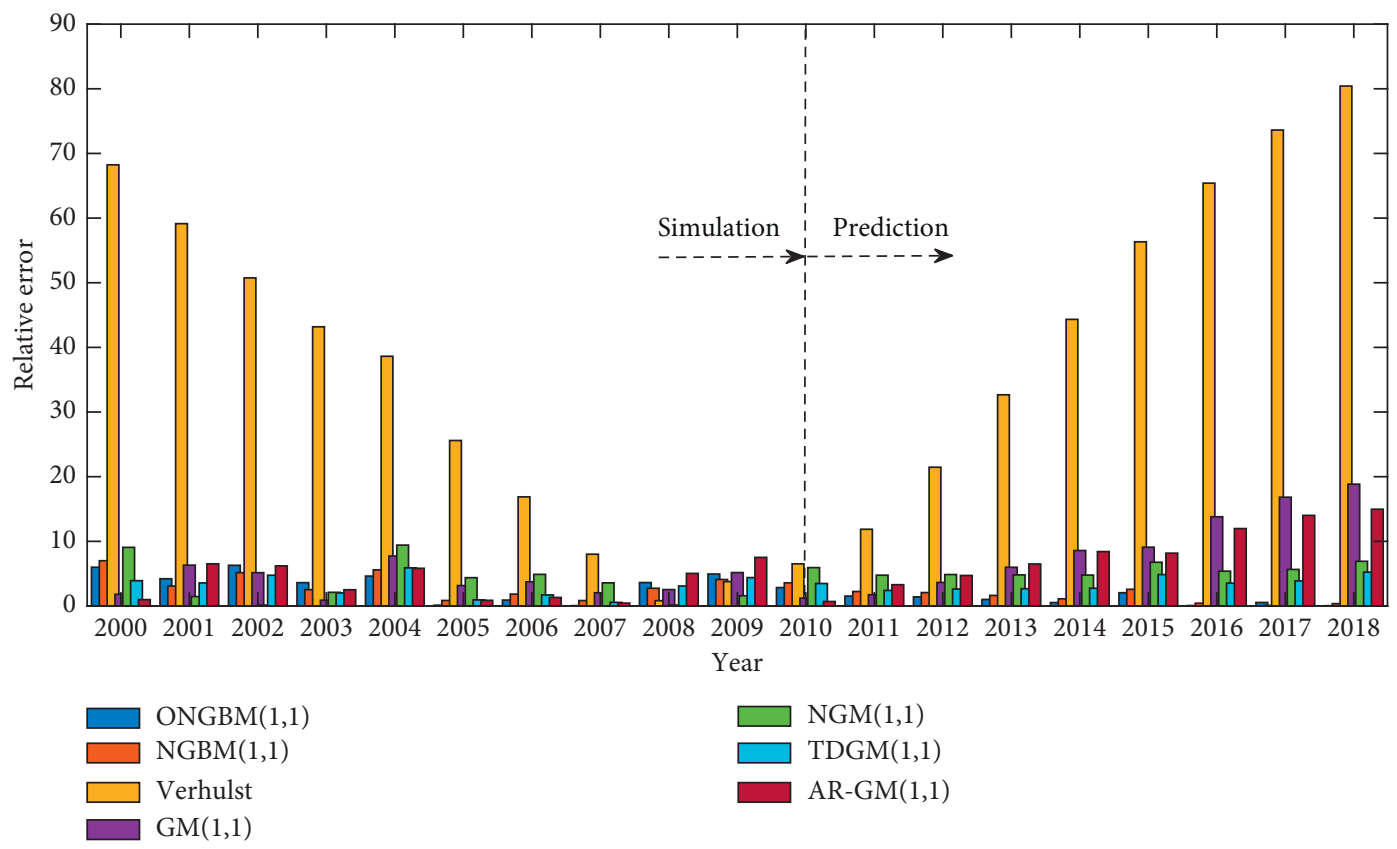

Figure 1: Overall APE comparison of seven models in Case 1.

process of the $\operatorname{ONGBM}(1,1)$ model is implemented in accordance with the steps described in Section 2.2.

3.1. Model Evaluation Indexes. The model evaluation indexes adopt the APE and MAPE that are commonly used in the grey model, and the specific calculation formulas are shown as equations (12) and (13). In the comparison process, the smaller the calculated values of APE and MAPE, the better the model effect. The calculation of MAPE is divided into two parts in this study, including MAPE $_{\mathrm{FIT}}$ of the fitting part and $\mathrm{MAPE}_{\mathrm{PRE}}$ of the prediction part. These two evaluation indexes should be comprehensively considered in the evaluation process of the model.

$$
\begin{aligned}
\operatorname{APE} & =\left(\frac{x^{(0)}(k)-\hat{x}^{(0)}(k)}{x^{(0)}(k)}\right) \times 100 \%, \quad k=1,2,3, \ldots, n . \\
\mathrm{MAPE} & =\frac{1}{n-1}\left(\sum_{i=1}^{n}\left|\frac{x^{(0)}(k)-\hat{x}^{(0)}(k)}{x^{(0)}(k)}\right|\right) \times 100 \%, \quad k=1,2,3, \ldots, n .
\end{aligned}
$$

3.2. Case Analysis. In order to illustrate the effectiveness of the model, this section applies the optimized $\operatorname{NGBM}(1,1)$ model to the oil data of four countries, respectively, and verifies the effectiveness of the model from different regions, which included the annual oil consumption data for China, Chile, Mexico, and Japan, and the data based on the BP Statistical Review of World Energy.

3.2.1. Case 1: Annual Oil Consumption of China. China is the most populous developing country and the second largest economy in the world. With the rapid development of China, the social productivity of China was significantly enhanced, and at the same time, the energy consumption ability also constantly improved, while nonfossil energy sources such as coal and oil are still the main energy sources in China currently. According to the data released by the National Bureau of Statistics of China, during the development process of China, there has been an obvious gap between the value of energy produced and the value of energy consumption and there has been a situation of short supply. Therefore, part of the energy begins to rely on imports; among them, oil accounts for a large proportion of imported energy. Therefore, the security of oil supply and demand is a problem that China needs to consider in the current development process.

In this paper, the oil consumption data from 1999 to 2018 in China were selected for model building, in which the data from 1999 to 2010 were used to establish the model and train the optimal parameter values, and the optimal 


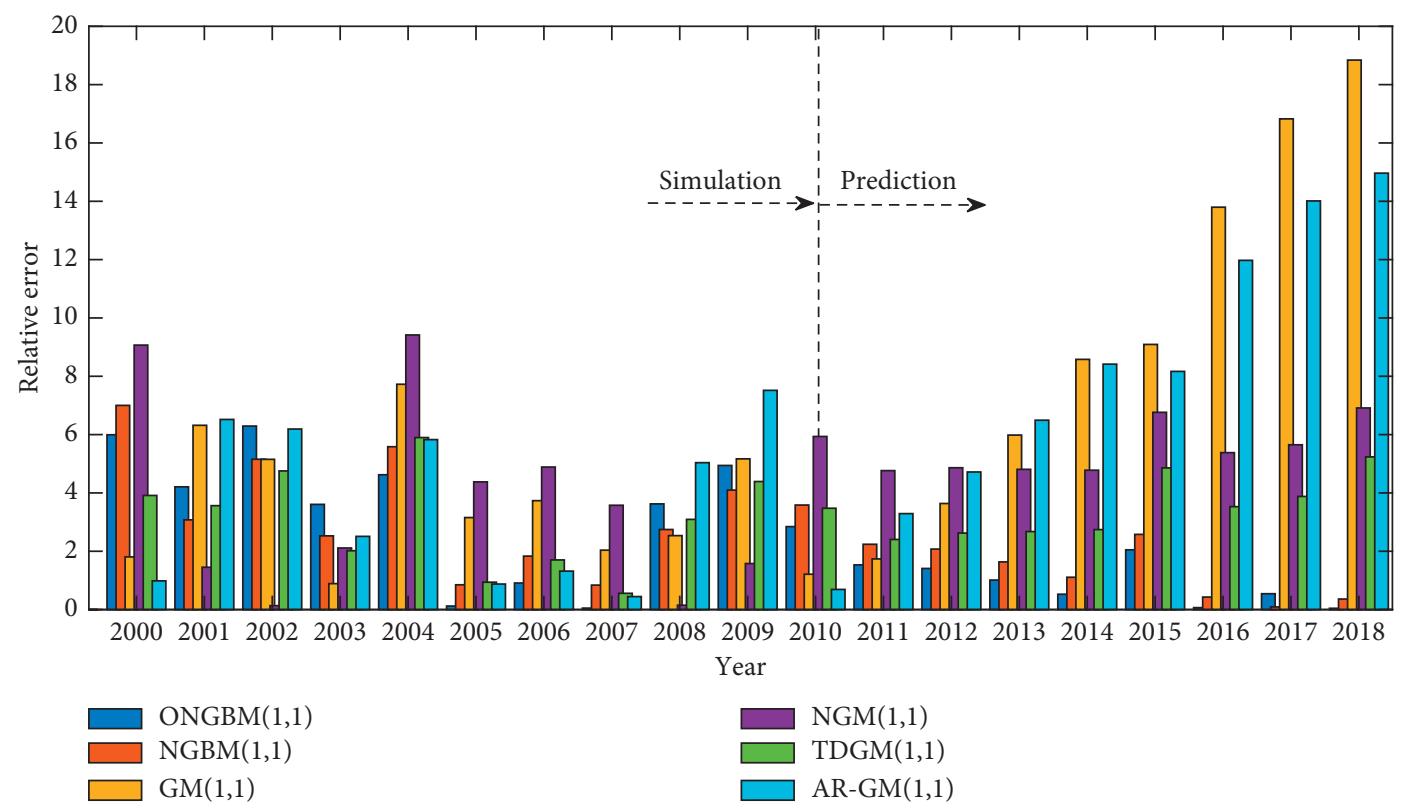

Figure 2: Overall APE comparison after removing the Verhulst model in Case 1.

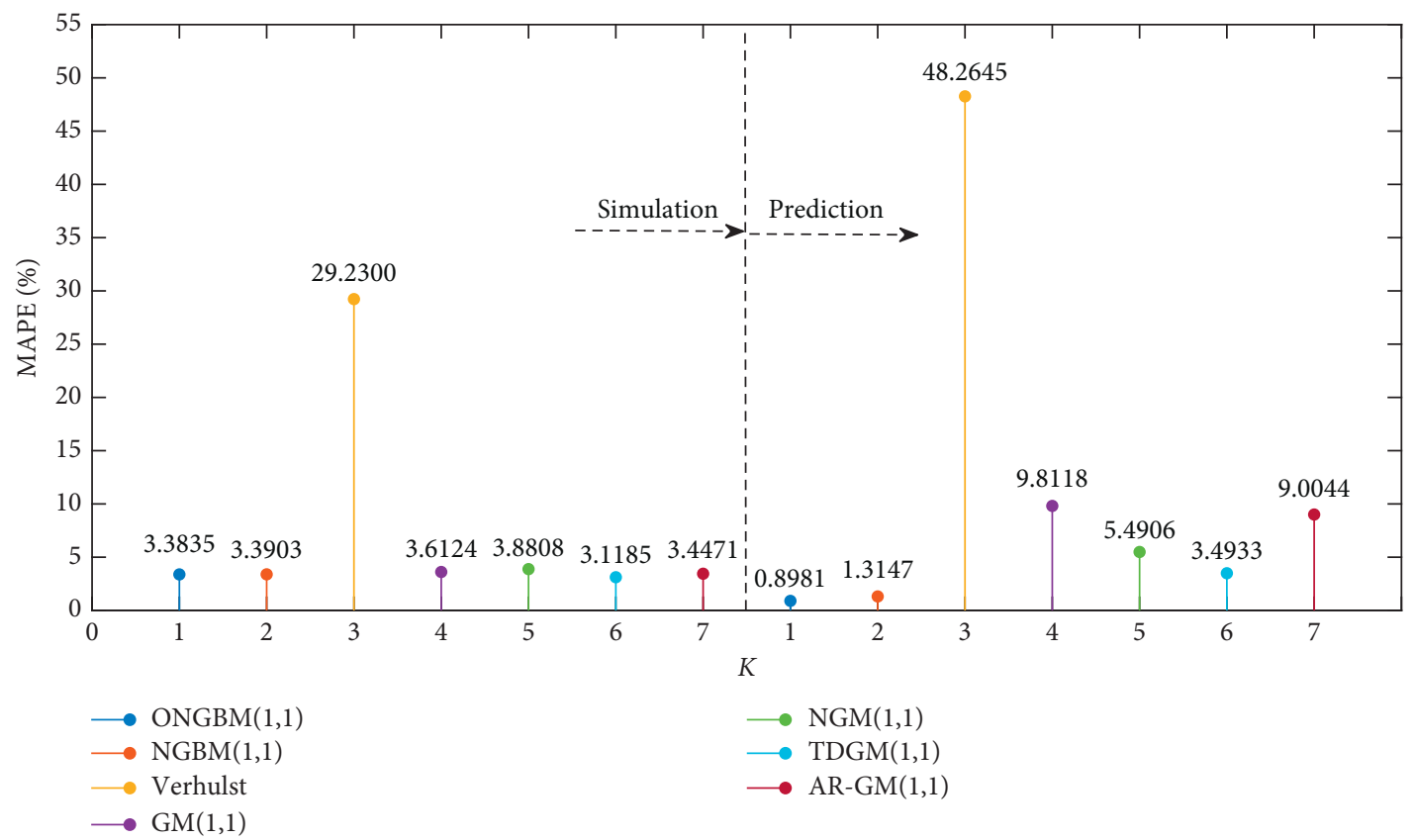

Figure 3: Comparison of MAPE in Case 1.

parameter values $r=0.174$ and $m=0.593$ are calculated. Then the data from 2011 to 2018 were used to verify the validity of the model. The simulation results and APE of the different models are shown in Table 1.

As can be seen in Table 1, the Verhulst model shows poor performance in both the simulation and prediction parts. Except for the Verhulst model, the remaining six models show no significant difference in the fitting part, and the APE all fluctuated at about 3.5\%. However, in the prediction part, although the prediction results of the $\operatorname{GM}(1,1)$, $\operatorname{NGM}(1,1)$, and AR-GM(1,1) models are all below $10 \%$, while they are higher than 5\%, compared with the other three models, and the deviation is relatively obvious. Then among the $\operatorname{ONGBM}(1,1), \operatorname{NGBM}(1,1)$, and $\operatorname{TDGM}(1,1)$ models, the accuracy of the optimized $\operatorname{NGBM}(1,1)$ model is higher, and its $\mathrm{MAPE}_{\mathrm{PRE}}$ reaches $0.8981 \%$, less than $1 \%$, and that compared with the unoptimized model, the original prediction accuracy is improved by $0.4166 \%$.

Figure 1 shows the APE comparison chart in Case 1. It can be clearly seen that the Verhulst model shows poor performance in the application of these data, so the results of the Verhulst model have a greater impact on the overall 


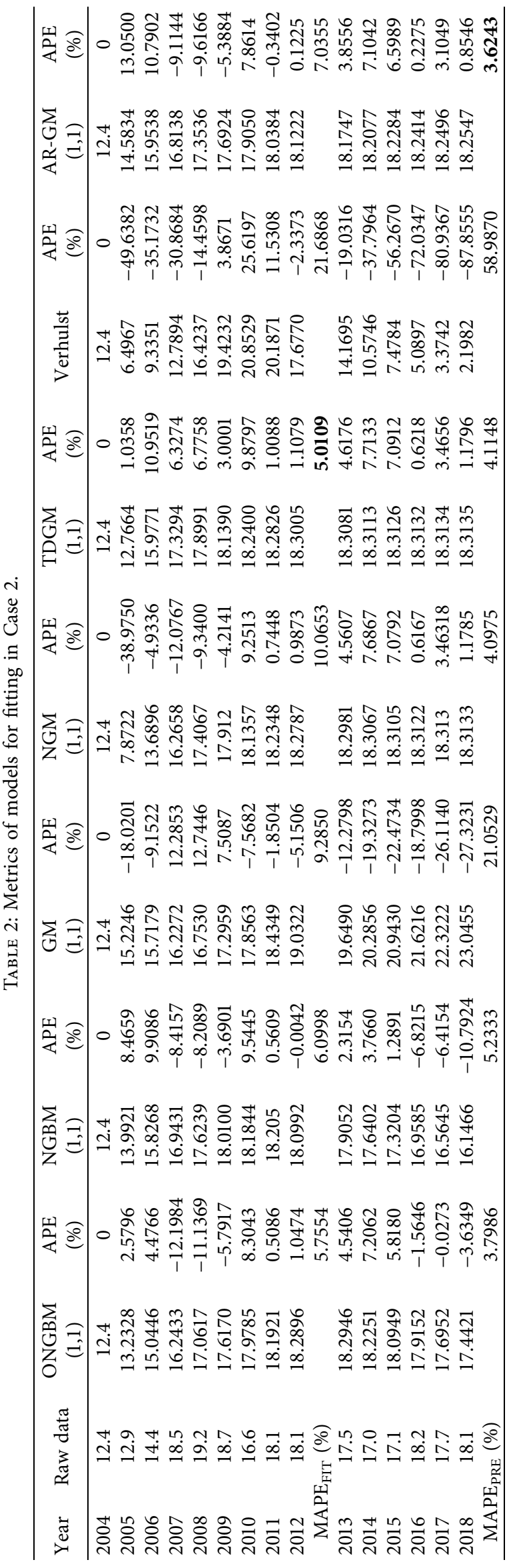




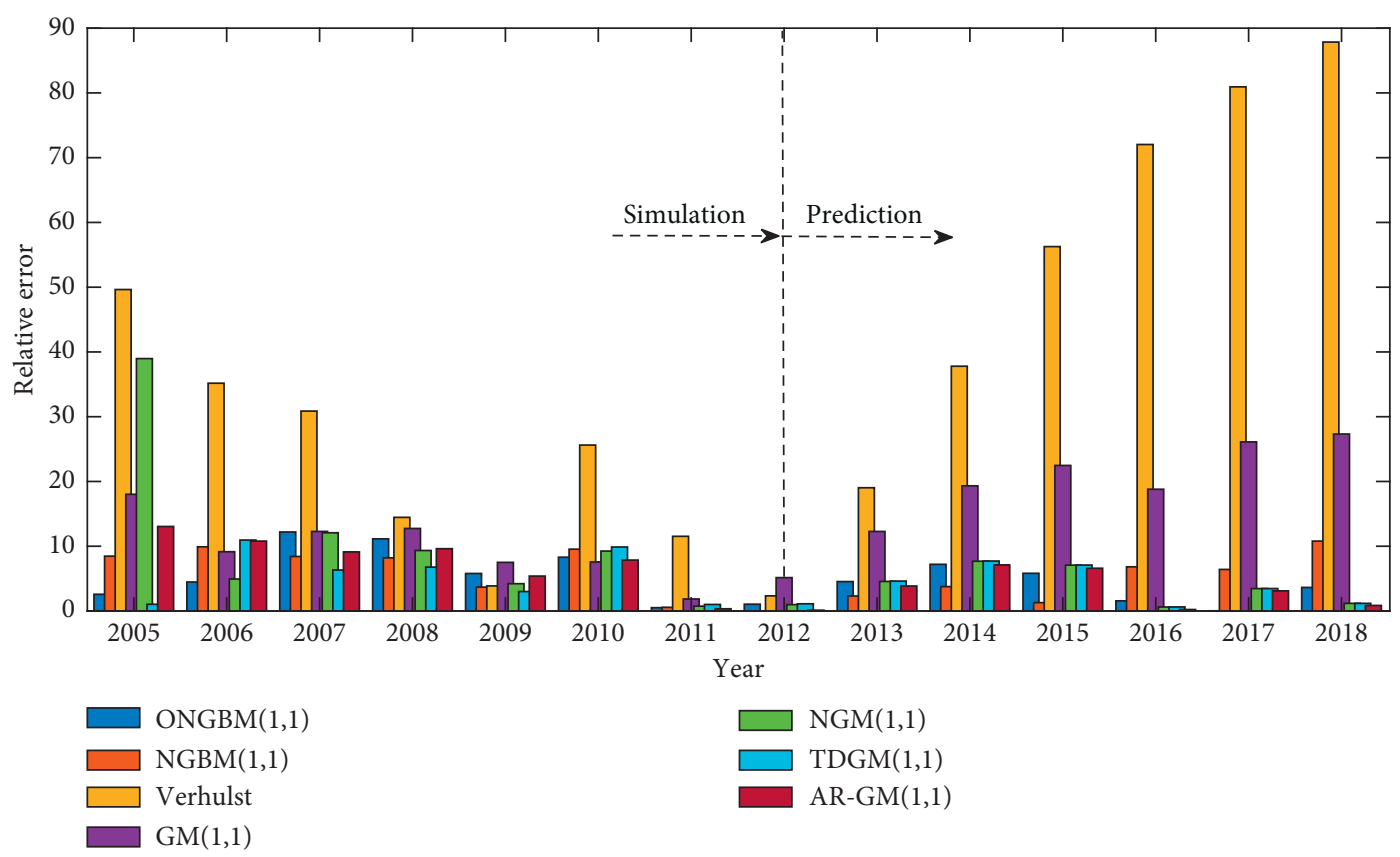

FIgURE 4: Overall APE comparison of seven models in Case 2.

comparison effect of all models. Then the comparison chart that removed the calculated data of the Verhulst model is drawn as shown in Figure 2.

In Figure 2, it can be seen that the relative error in the prediction part fluctuates within a small range, among which the $\operatorname{NGM}(1,1)$ model has a few large deviations at some points. However, the overall relative error of $\operatorname{NGM}(1,1)$ of both simulation and prediction parts is in a relatively stable state. In the prediction part shown in Figure 2, it can also be seen that the relative errors of $\operatorname{GM}(1,1)$ and $\operatorname{AR}-\operatorname{RM}(1,1)$ models show a trend of a gradual rise, and the deviation is gradually larger.

Figure 3 shows the MAPE comparison of the seven models in Case 1, which can more intuitively see the performance difference between different models. The overall level of the fitting part is similar except for the Verhulst model while the ONGBM $(1,1)$ model has more advantages in the prediction part.

3.2.2. Case 2: Annual Oil Consumption of Chile. Chile is the longest and narrowest country in the world, which is rich in mineral resources, but short of energy. The production of coal, oil, and natural gas in Chile is low and mainly depends on imports from other countries. Chile is not a populous country, and thus, the import volume will fluctuate greatly with the demand of the country. Therefore, it can be seen from Table 2 that the annual oil consumption data of Chile fluctuated to a certain extent. In this case, the oil consumption data of Chile from 2004 to 2018 were selected for analysis. The original data and the calculated data of seven models are shown in Table 2. The data from 2004 to 2012 were used to establish the model, and the data from 2013 to 2018 were used to test the validity of the model.
It can be seen from the calculation results in Table 2 that, in the fitting part, the effects of $\operatorname{ONGBM}(1,1), \operatorname{NGBM}(1,1)$, and $\operatorname{TDGM}(1,1)$ are closer, and the effect of $\operatorname{TDGM}(1,1)$ is slightly better than that of the optimized NGBM $(1,1)$ model, and the accuracy of $\operatorname{ONGBM}(1,1)$ is $0.3444 \%$ higher than that of the unoptimized $\operatorname{NGBM}(1,1)$ model. In the prediction part, the AR-GM $(1,1)$ model has the best effect, and compared with ONGBM $(1,1)$ model, the MAPE $\mathrm{PRE}_{\mathrm{P}}$ of the AR$\operatorname{GM}(1,1)$ model was $0.1743 \%$ higher, but the fitting error of the AR-GM(1,1) model is relatively higher than that of ONGBM $(1,1)$ model. Although the $\mathrm{MAPE}_{\mathrm{FIT}}$ and MAPE $\mathrm{PRE}$ of the ONGBM $(1,1)$ model are not optimal in this case, the comprehensive consideration shows that the $\operatorname{ONGBM}(1,1)$ model still has certain desirability, and the $r=0.287$ and $m=0$ can be calculated in this case.

In the APE comparison shown in Figure 4, it can be seen that the APE of the Verhulst model in the prediction part is obviously larger than that of other models, and from the calculation results of Table 3 , it can be known that its MAPE $_{\text {PRE }}$ has reached $58.9870 \%$, which led the selection of the error interval too large in the process of mapping and then affected the comparison effect of other models. Therefore, in this case, the relative error value of the Verhulst model is removed and the APE comparison chart is redrawn, as shown in Figure 5.

It can be seen in Figure 5 that the fitting error of the $\operatorname{NGM}(1,1)$ model in 2005 was obviously higher, and there was an abnormality at one point, and then, the subsequent nodes tended to be stable. In the fitting part, before 2010, the errors of the seven models were relatively obvious, and the fluctuation range was relatively large. After 2011, except for the $\operatorname{GM}(1,1)$ and $\operatorname{NGBM}(1,1)$ models, the relative errors of the other models showed no obvious abnormal changes and were relatively stable. 


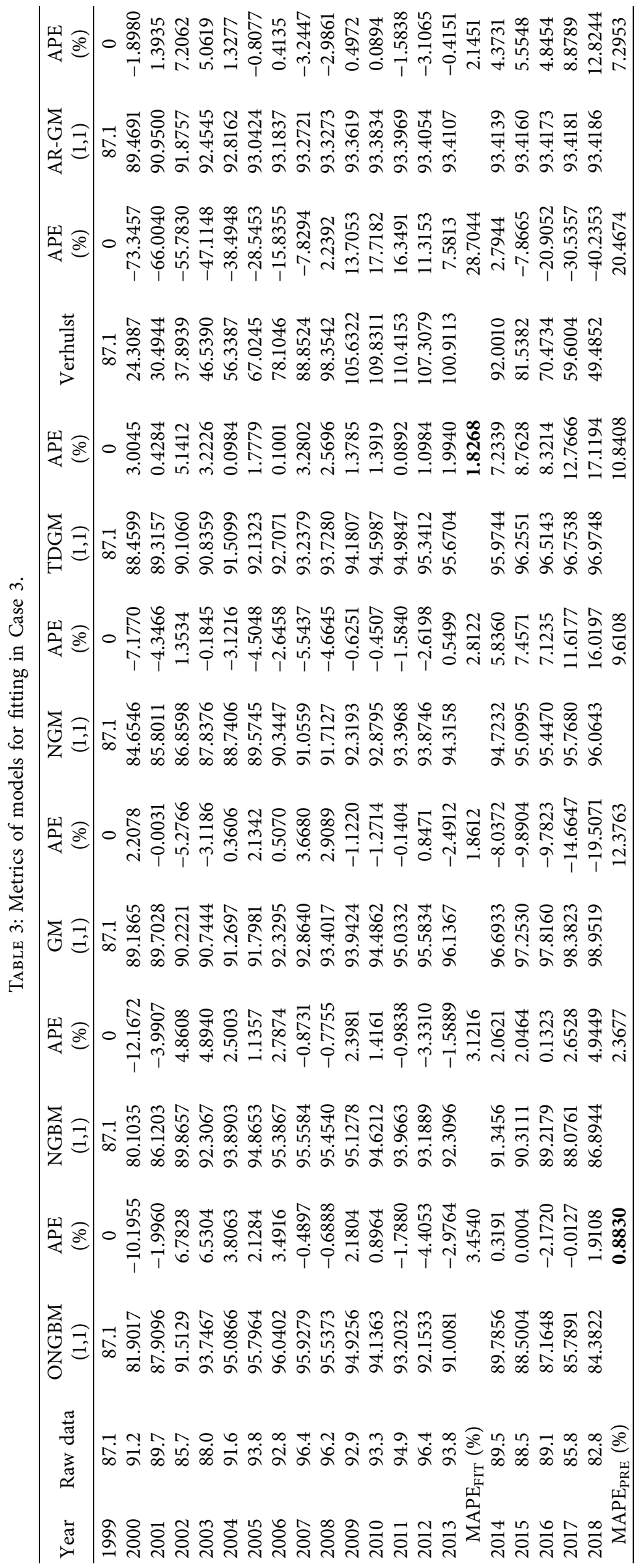




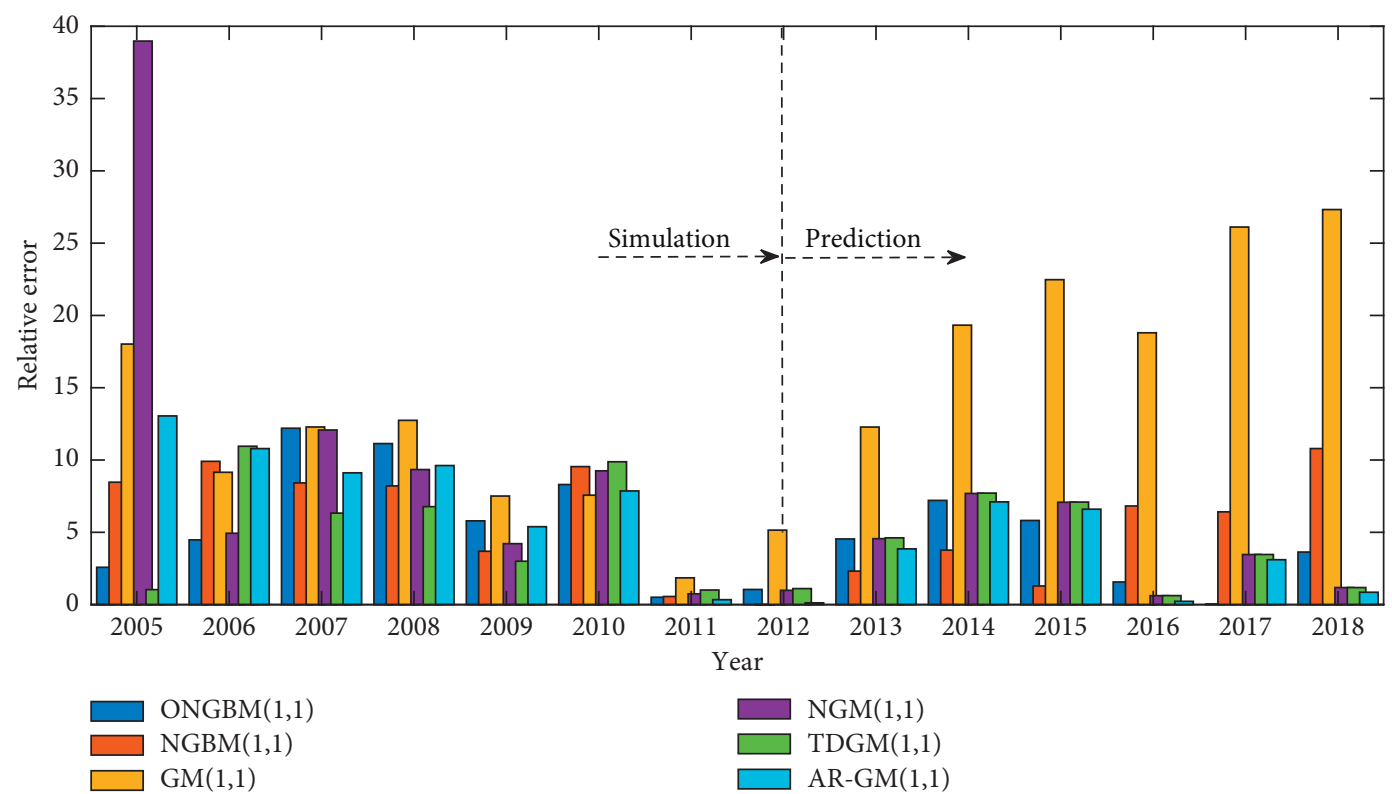

Figure 5: Overall APE comparison after removing the Verhulst model in Case 2.

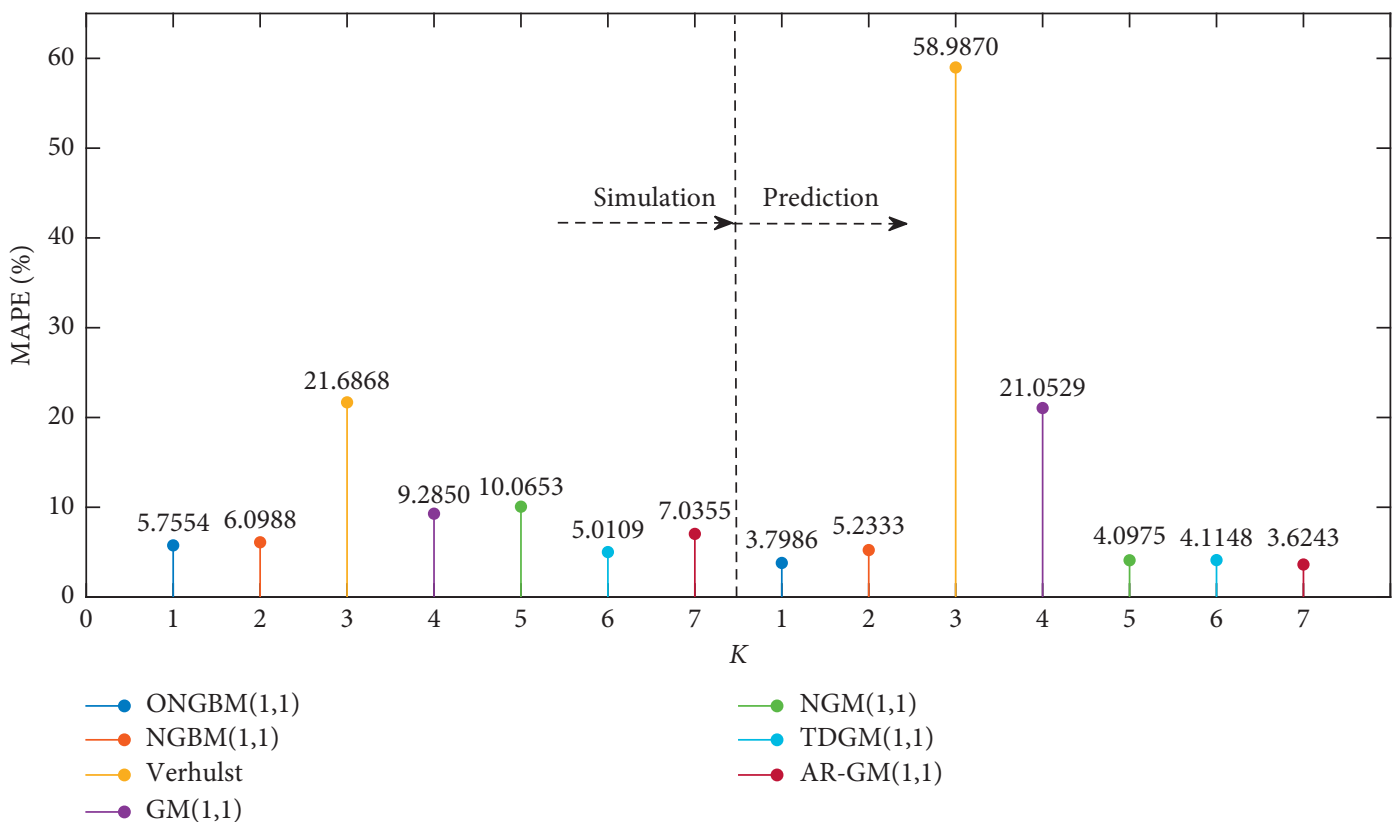

Figure 6: Comparison of MAPE in Case 2.

In order to see the MAPE comparison of the different models in Table 2 intuitively, Figure 6 presents the MAPE comparison diagram of the simulation part and prediction part, through that the comparison effect is more obvious.

3.2.3. Case 3: Annual Oil Consumption of Mexico. Mexico is rich in oil and gas resources and is one of the largest oil producers in the world. EIA data show that the crude oil production of Mexico has shown a downward trend since 2010, and corresponding to the "BP Statistical Review of World Energy" data released by $\mathrm{BP}$, it can be seen that the oil consumption data of Mexico have shown a gradual decrease since 2007. This paper selects the oil consumption data of Mexico from 1999 to 2018. The original data of Mexico's annual oil consumption and the simulation data of the $\operatorname{ONGBM}(1,1)$ model and the other comparison models are given in Table 1. Then, the data from 1999 to 2013 are used to build the model and calculate the model parameters, and the data from 2014 to 2018 are used to test the validity of the model.

The oil consumption data of Mexico are substituted into the model, and the optimal parameters of the $\operatorname{ONGBM}(1,1)$ model are calculated by the simulated annealing algorithm, and then $r=0.185$ and $m=0.939$. From the calculation results of the 


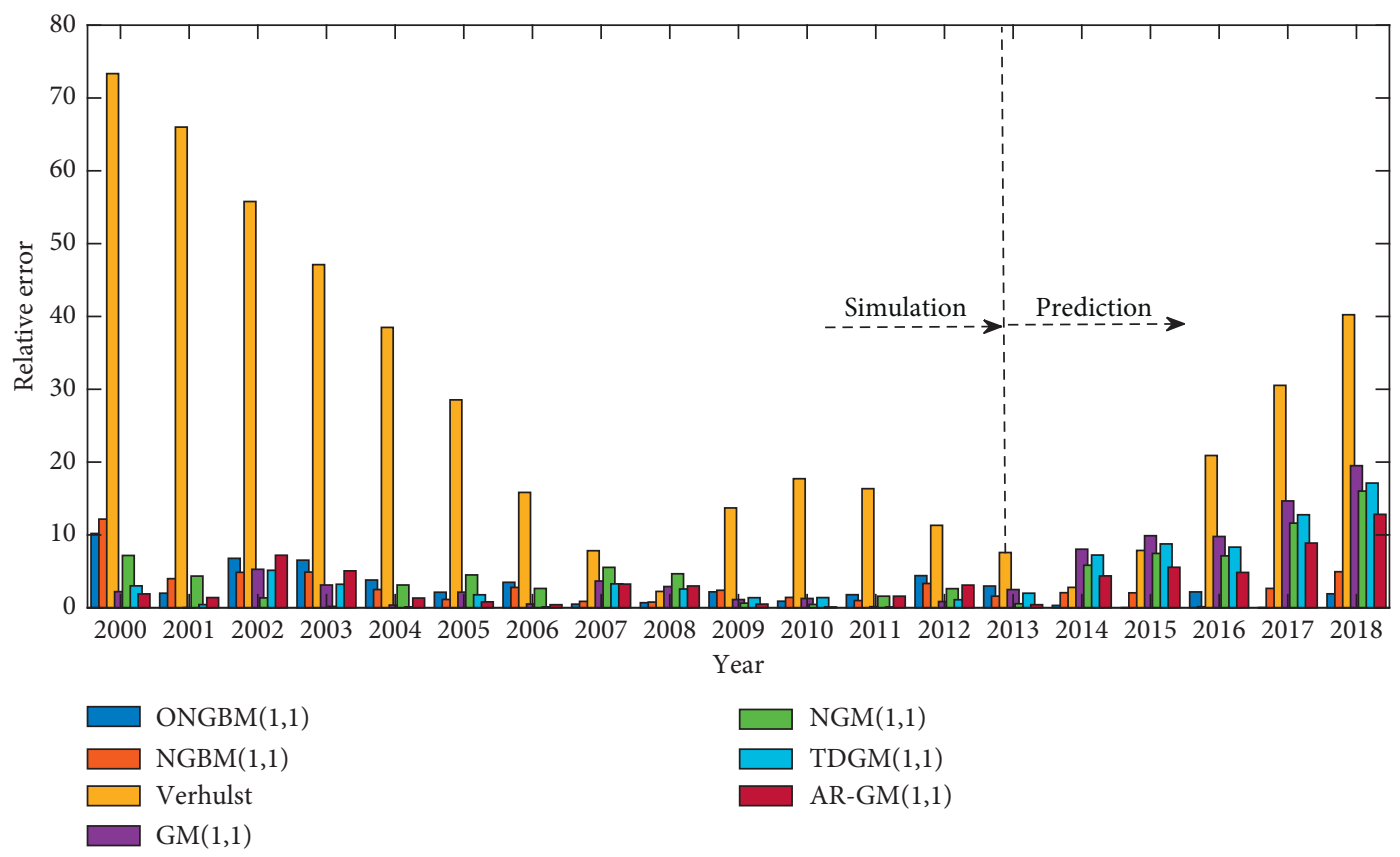

Figure 7: Overall APE comparison of seven models in Case 3.

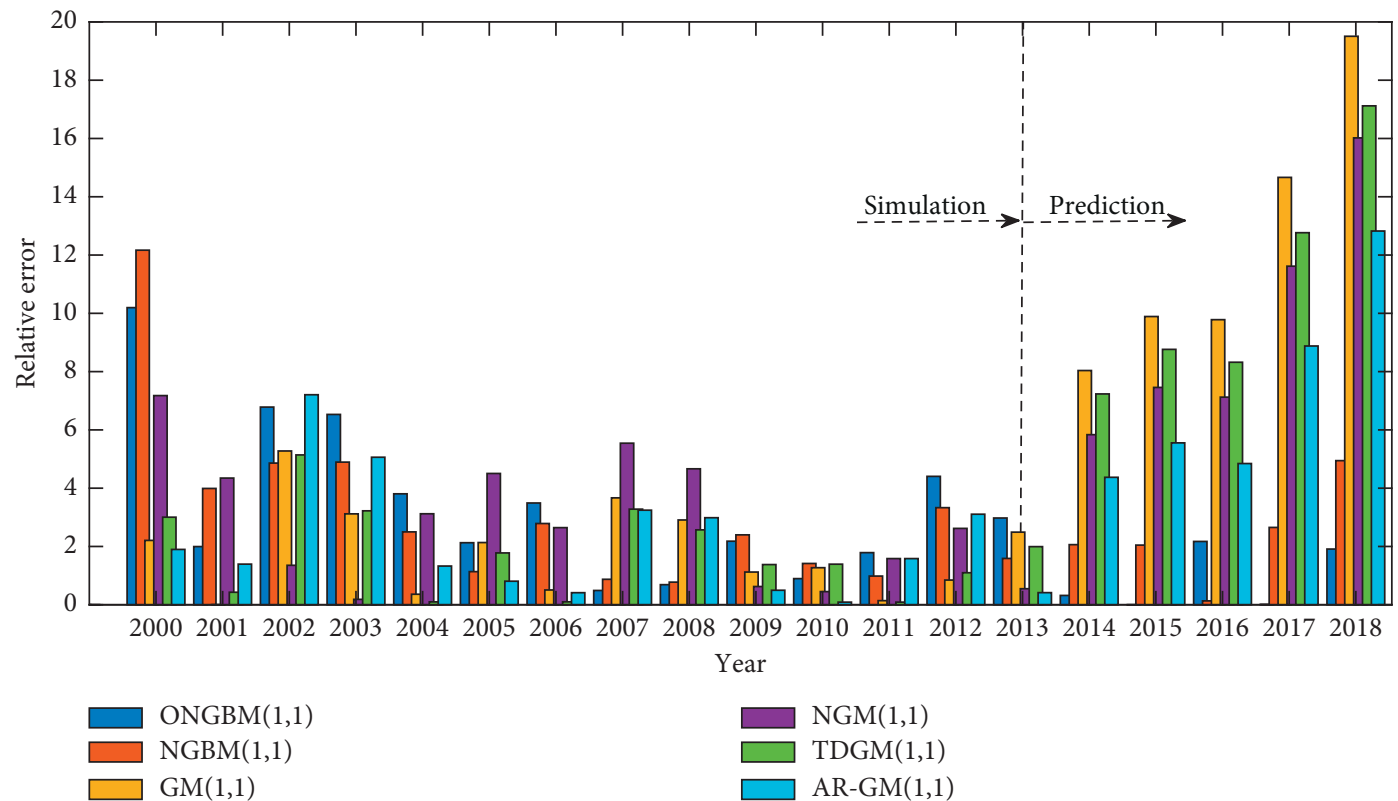

Figure 8: Overall APE comparison after removing the Verhulst model in Case 3.

ONGBM $(1,1)$ model and the other six comparison models in Table 1, it can be seen that, except for the Verhulst model, all other models exhibit higher accuracy in the fitting process, among which the MAPE $_{\mathrm{FIT}}$ of the $\operatorname{GM}(1,1), \operatorname{NGM}(1,1)$, $\operatorname{TDGM}(1,1)$, and AR-GM(1,1) model are all lower than $3 \%$, and there was no significant difference between them, and it is slightly higher than that of the $\operatorname{ONGBM}(1,1)$ and $\operatorname{NGBM}(1,1)$ models in the process of fitting. And the best performance in the simulation part is shown by the $\operatorname{TDGM}(1,1)$ model, whose $\mathrm{MAPE}_{\mathrm{FIT}}$ reaches $1.8268 \%$. In the prediction part, the Verhulst model also shows poor results compared with other models, while $\operatorname{GM}(1,1)$ and $\operatorname{TDGM}(1,1)$ models showed a slightly poor performance in the prediction part, with MAPE $_{\mathrm{PRE}}$ higher than $10 \%$, and the prediction errors of the $\operatorname{NGM}(1,1)$ and AR$\operatorname{GM}(1,1)$ models are below $10 \%$, while there is a slight deviation compared with the ONGBM $(1,1)$ model. The comparison between $\operatorname{ONGBM}(1,1)$ and $\operatorname{NGBM}(1,1)$ shows that the accuracy of the optimized model in the fitting effect is slightly increased by $0.3324 \%$, but the prediction error is significantly improved, which reaches less than $1 \%$, reflecting the better performance.

In order to see the comparison calculation results in Table 1 more intuitively, Figure 7 shows the APE 


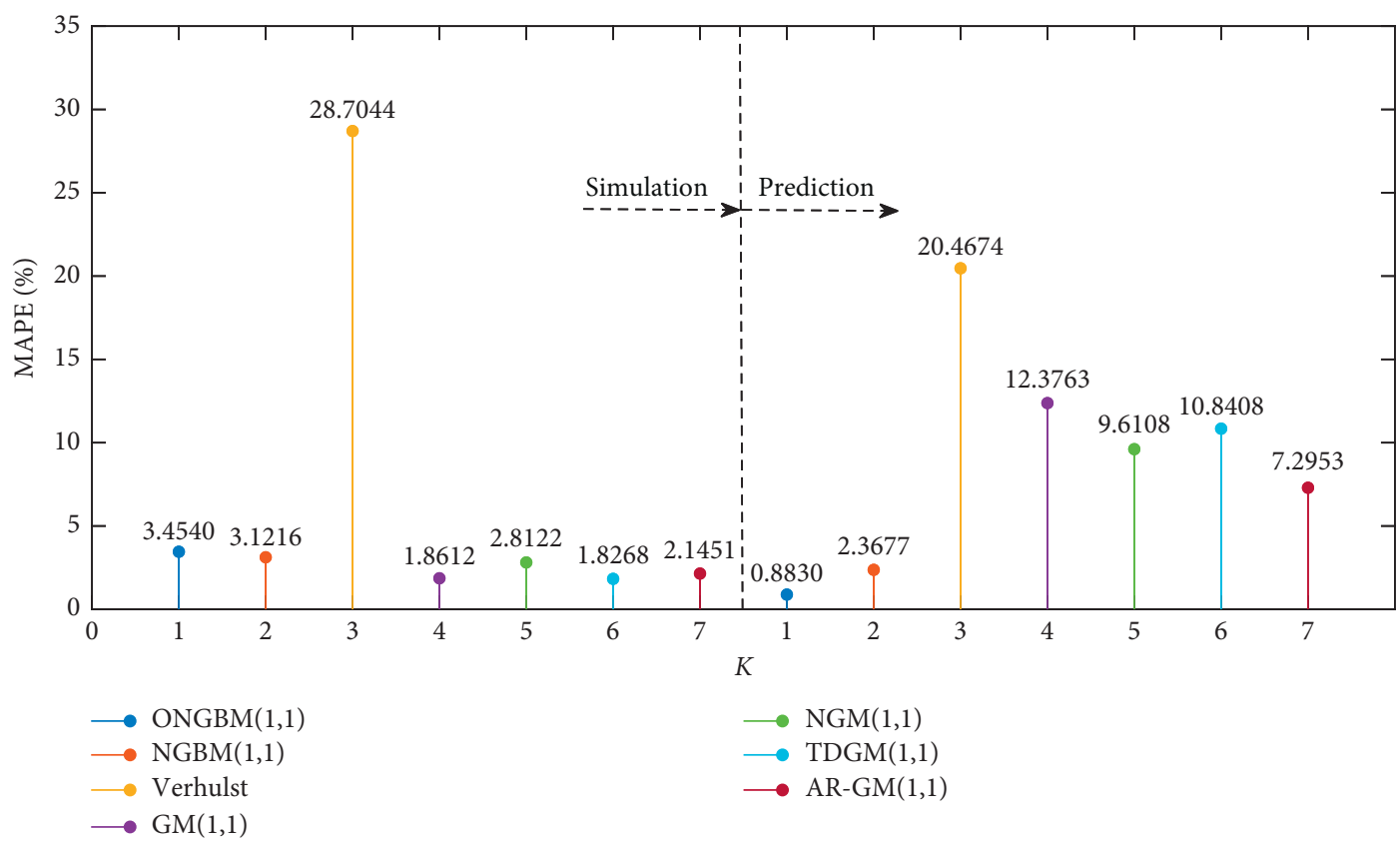

Figure 9: Comparison of MAPE in Case 3.

comparison diagram of seven models. It can be clearly seen that the deviation of the Verhulst model is very large, and the largest deviation of which reached more than $70 \%$, because the error of the Verhulst model is too large and the value of the vertical axis of the image is increased, making the contrast effect of other models not obvious, and therefore, Figure 8 shows the contrast effect after removing the Verhulst model.

It can be seen from Figure 7 that the fitting errors of the first four points of the $\operatorname{ONGBM}(1,1)$ model fluctuate greatly in the fitting part and tend to be stable in the subsequent nodes. In the fitting part, the relative errors of most nodes of the seven models fluctuate less than $5 \%$ after the fifth time node. In the prediction part, the APE of GM $(1,1)$ was significantly increased by node, while the relative error of $\operatorname{ONGM}(1,1)$ and $\operatorname{ONGBM}(1,1)$ models fluctuated around $2 \%$ in this part, and $\operatorname{ONGBM}(1,1)$ showed a better effect.

The comparison of the MAPE values of the seven models in the simulation and prediction part is shown in Figure 9, and the effects of each model can be clearly distinguished from the figure.

3.2.4. Case 4: Annual Oil Consumption in Japan. Japan is a highly developed capitalist country, which is located on an island in East Asia. Because the domestic resources in Japan are relatively scarce and the proven oil reserves are limited, its energy is mainly dependent on imports. However, as the third largest economy in the world, Japan has become the world's fifth largest oil consumer after the United States, China, India, and Russia. Japan is also the fourth largest crude oil importer after the United States, China, and India. Therefore, the analysis and forecast of oil consumption in Japan are particularly important. According to the data from "BP Statistical Review of World Energy," annual oil consumption of Japan has shown a downward trend since 2012, which is closely related to the aging of the country's internal population, the decline of the population, and the improvement of energy efficiency by Japanese domestic technology. This case selects the data from 2008 to 2018 to build the model and evaluates the validity of the model. The data from 2008 to 2012 are used to build the model, and the data from 2013 to 2018 are used to verify the validity of the model. The original data and simulation data of the seven models are shown in Table 4.

From the results shown in Table 4, it can be observed that the $\operatorname{NGM}(1,1)$ model has the largest deviation in the fitting part. The ONGBM $(1,1), \operatorname{NGBM}(1,1), \operatorname{GM}(1,1)$, and $\operatorname{AR}-\operatorname{GM}(1,1)$ models have similar performance effects, but the effect of $\operatorname{ONGBM}(1,1)$ is slightly better than the other three models. Substituting the data in Case 4 into the Verhulst model, it can be found that the fitting part showed good results. The MAPE value was less than $10 \%$, but its prediction error was about $65 \%$, and the prediction performance was poor. In fact, except for the ONGBM $(1,1)$ model and $\operatorname{NGBM}(1,1)$ model, the prediction error of other models exceeds $10 \%$. But on the whole, the prediction effect of the optimized $\operatorname{NGBM}(1,1)$ model has been significantly improved, and the $\mathrm{MAPE}_{\mathrm{FIT}}$ value has increased by $2.997 \%$. The parameters corresponding to the optimized $\operatorname{ONGBM}(1,1)$ are $r=0.175$ and $m=0.773$.

It can be seen from Figure 10 that, except for the large deviation of the Verhulst and the $\operatorname{NGM}(1,1)$ model in 2010, the relative error of the other years presents a relatively stable state. In fact, it can be known from the calculation results of Table 4 that the MAPE $\mathrm{PRE}_{\text {of }}$ of the Verhulst model reached $68.4851 \%$. It can also be seen in the comparison chart of Figure 10 that in the prediction part, the relative error value of the Verhulst model shows a gradually increasing trend. Because the error calculated by it is too large and affects the comparison effect of other models, the comparison result 


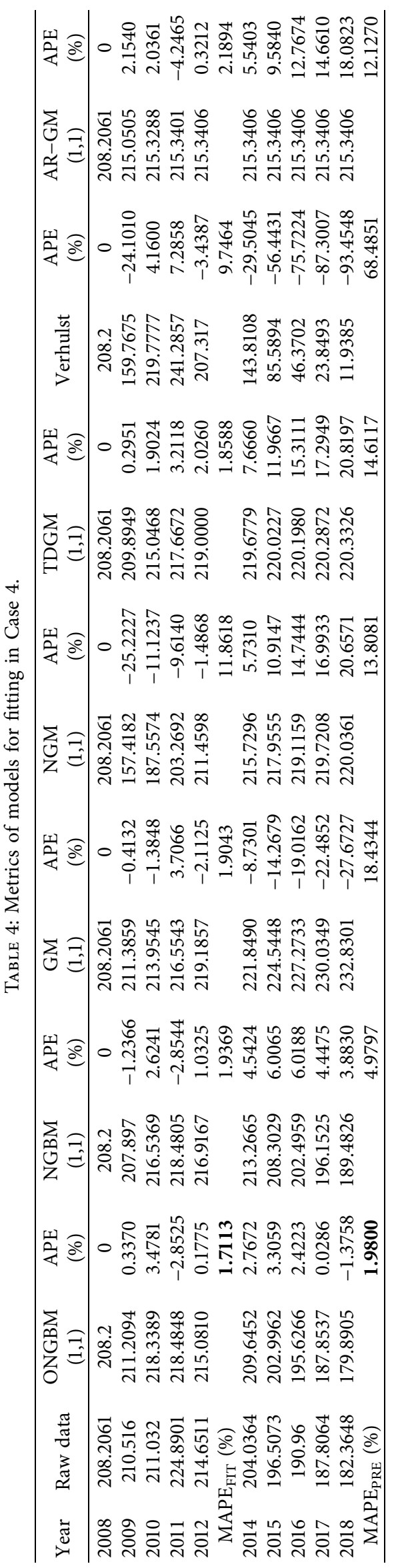




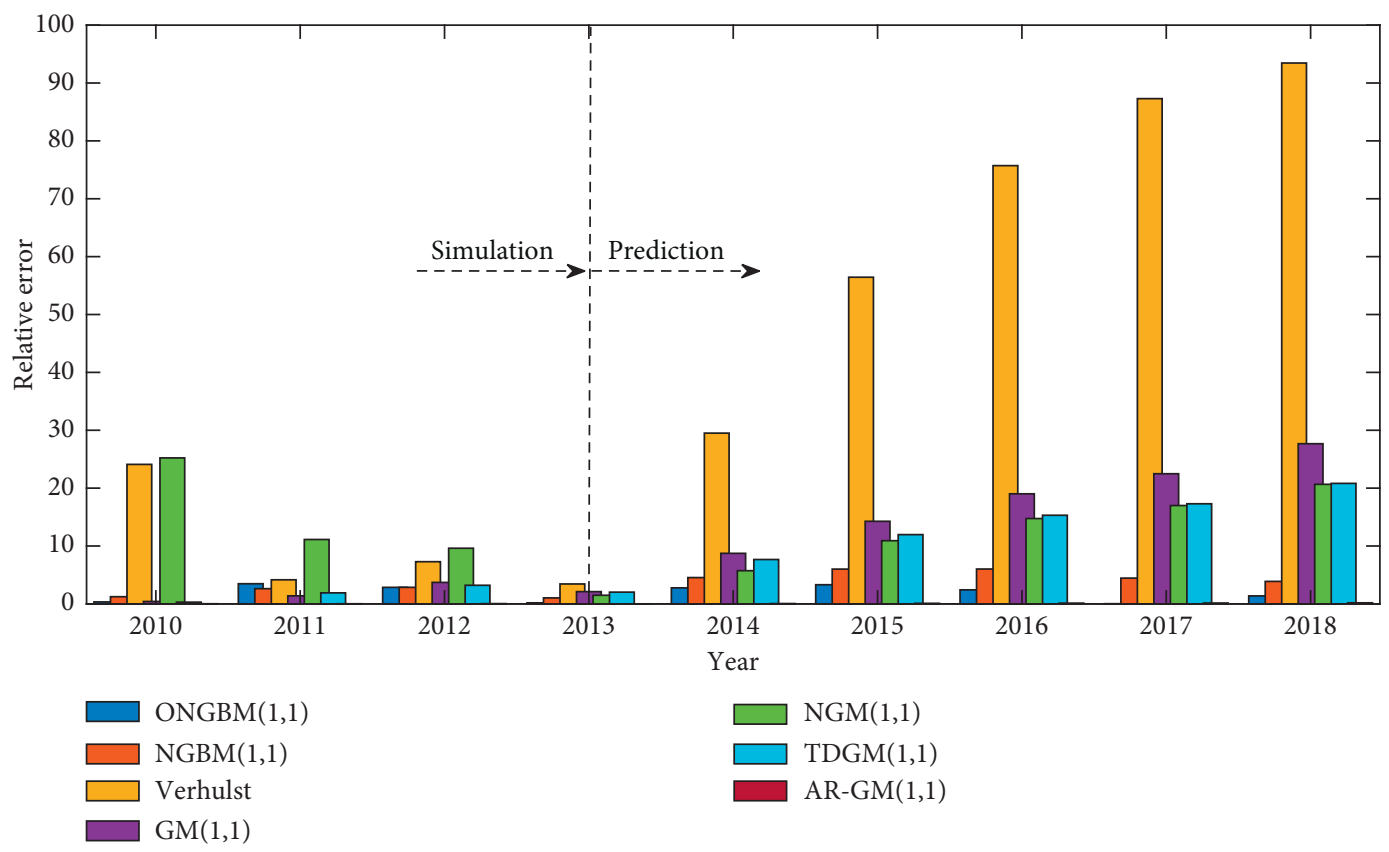

FIgure 10: Overall APE comparison of seven models in Case 4.

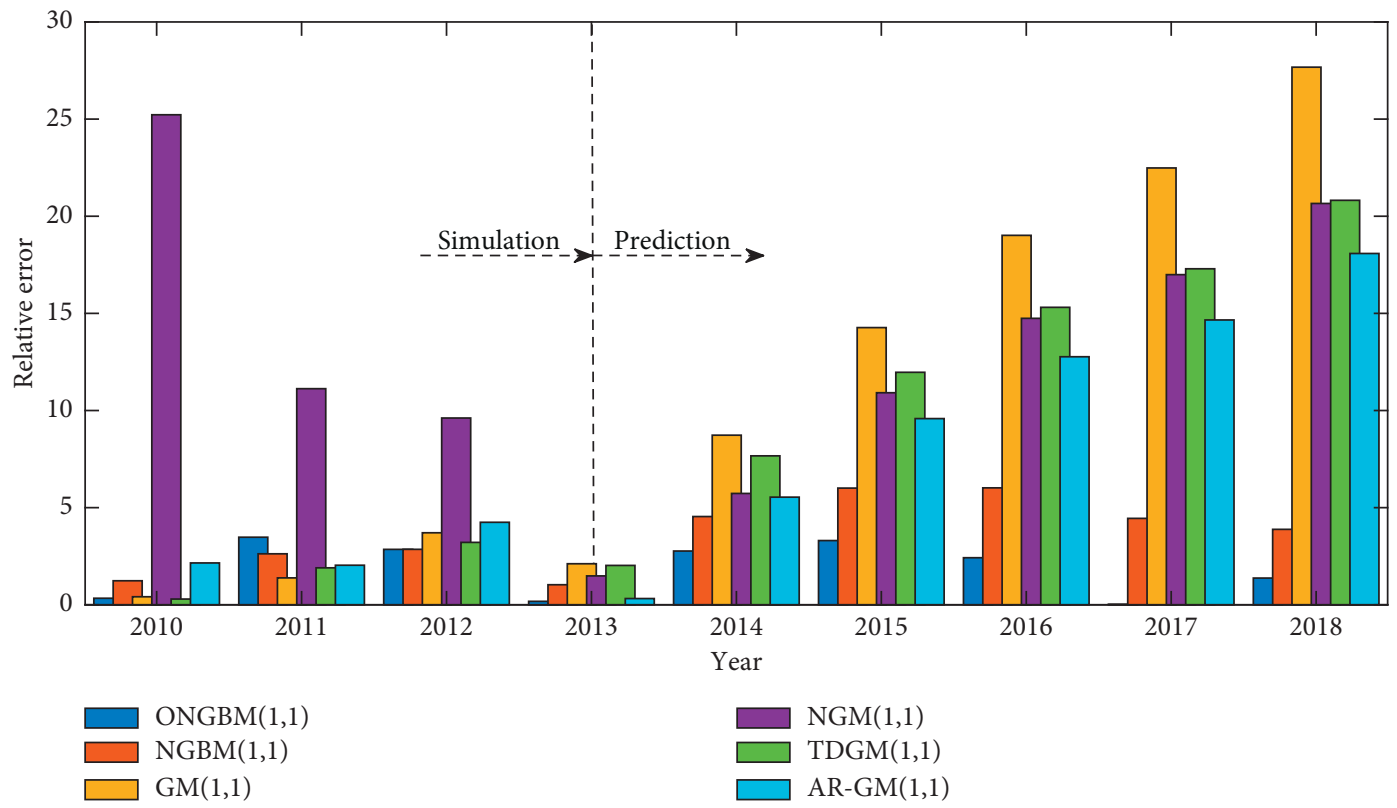

FIgURe 11: Overall APE comparison after removing the Verhulst model in Case 4.

after removing the relative error data of the Verhulst model is shown in Figure 11.

In the APE comparison chart shown in Figure 11, it can be clearly seen that the relative errors calculated by the $\operatorname{GM}(1,1), \operatorname{NGM}(1,1), \operatorname{TDGM}(1,1)$, and $\operatorname{AR}-\operatorname{GM}(1,1)$ models are gradually increasing in the prediction part, and each model showed the best performance in 2013 . Moreover, the $\operatorname{ONGBM}(1,1)$ model is indeed better than other models.

The comparison of the MAPE values of the seven models in the simulation and prediction part is shown in Figure 12, and the effects of each model can be clearly distinguished from the figure.

\section{Application of the ONGBM(1,1) Model}

Based on the modelling analysis in Section 3.2, this paper predicts the oil consumption of the above four countries in the next five years, and the forecast data are shown in Table 5.

It can be analyzed from the forecast results in Table 5 that the annual oil consumption of most countries in the next five years will still show a continuous downward trend. However, 


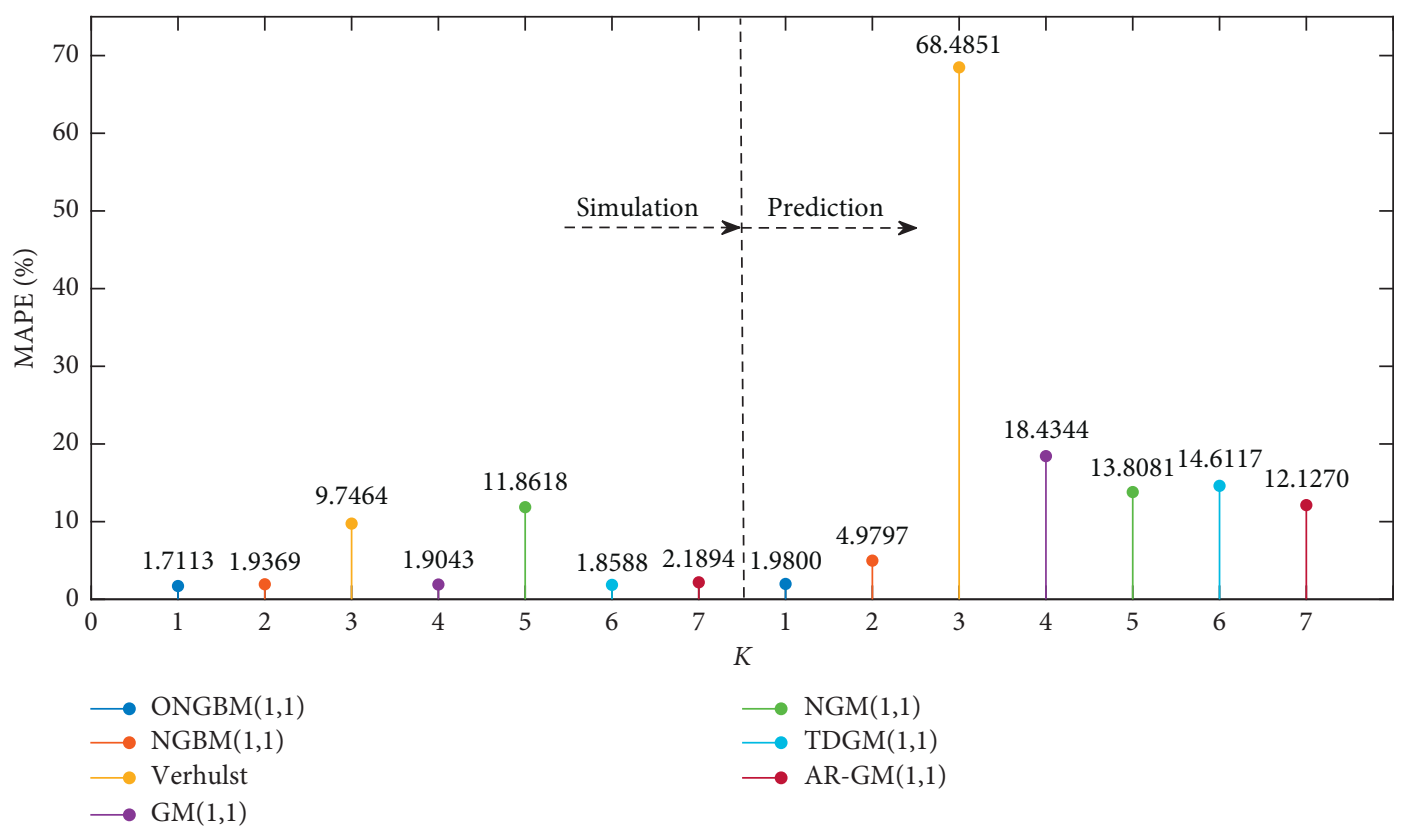

Figure 12: Comparison of MAPE in Case 4.

TABLE 5: Forecast results of oil consumption in the four countries in the next 5 years.

\begin{tabular}{|c|c|c|c|c|}
\hline \multirow{2}{*}{ Year } & \multicolumn{4}{|c|}{ Country } \\
\hline & Mexico & Chile & China & Japan \\
\hline 2019 & 82.9514 & 17.1622 & 669.8260 & 164.3109 \\
\hline 2020 & 81.5033 & 16.8605 & 699.0912 & 155.9098 \\
\hline 2021 & 80.0434 & 16.5415 & 729.3230 & 147.7182 \\
\hline 2022 & 78.5763 & 16.2089 & 760.5658 & 139.7819 \\
\hline 2023 & 77.1063 & 15.8656 & 792.8642 & 132.1313 \\
\hline
\end{tabular}

China is the most populous country in the world, which is in a stage of full-speed development currently. Although it has to take the initiative to adjust its energy structure, due to its large population, vast areas of economic development, and too many related factors, it will often be implemented after the policy is implemented. At the same time, since coal is the first energy source in China, reducing the dependence on coal is a major goal of China. Therefore, the consumption of oil as a substitute for coal may still increase; Chile is an energy-scarce country; in recent years, as the world gradually began to adjust its state of energy structure, Chile also established its own policy because Chile belongs to small population, and thus, the implementation of measures can see the effect relatively quickly. Therefore, the country may have a downward trend in its annual oil consumption under the current environment of energy restructuring. The oil production of Mexico has been declining since 2010, and its annual oil consumption has been declining year by year since 2007; the resources in Japan are relatively scarce, and it mainly depends on imports, and the data show that the oil consumption of Japan has shown a downward trend since 2012. Based on the above reasons and the forecast results of annual oil consumption in the next five years, it can be concluded that oil consumption is on a downward trend in most countries.

\section{Conclusion}

This paper optimizes the background value of the $\operatorname{NGBM}(1,1)$ model and proposes the $\operatorname{ONGBM}(1,1)$ model on this basis, and the simulated annealing algorithm was used to optimize undetermined coefficients in the $\operatorname{NGBM}(1,1)$ model. Actual oil consumption data from four countries, China, Chile, Mexico, and Japan, were used to test the effectiveness of the model. The results show that the optimized $\operatorname{ONGBM}(1,1)$ model is basically better than the other six grey prediction models. At the same time, applying the model to the oil consumption forecasts of the above four countries, it can be concluded that the oil consumption of the other three countries is showing a downward trend, except for China. This phenomenon is related to different factors such as the development measures of the own country, geographic location, resource ownership, and the economic development status of the country.

In this paper, the background values of the $\operatorname{NGBM}(1,1)$ model were optimized considering the nonlinear structure of the energy system. The simulated annealing algorithm is used to find the optimal undetermined coefficients, and an optimized ONGBM $(1,1)$ model is established. From the two perspectives of modelling mechanism and data applicability, the actual oil consumption data of China, Chile, Mexico, and 
Japan were selected to analyze the validity of the model. The selected data contain three types, namely, the data with steady rise, the data with irregular fluctuation, and the data with gradual decline and fluctuation. The comparison results show that the new model has a good effect under the two indexes, and the effect of the optimized ONGBM $(1,1)$ model is basically better than that of the other six grey prediction models. Therefore, on the basis of the modelling analysis in Section 3.2, the model is continued to be applied to the oil consumption forecast of these four countries in 2019-2023. The forecast results show that, except China, the oil consumption of the other three countries shows a downward trend. This phenomenon is related to different factors such as the country's development measures, geographical location, resource availability, and national economic development.

The optimization model proposed in this paper mainly considers the development trend of the variable itself and then research and analysis on it. The nonlinear characteristic of the energy system is considered in the research, but the influence of other factors on energy development is rarely considered. To a certain extent, this problem restricts the application range of the model, so how to solve this problem better considering the influence of other factors on variables will be the focus of our future work.

\section{Data Availability}

The data used to support the findings of this study are available from the corresponding author upon request.

\section{Conflicts of Interest}

The authors declare that there are no conflicts of interest regarding the publication of this paper.

\section{References}

[1] J. L. Deng, Estimate and Decision of Grey System, Huazhong University of Science and Technology Press, Wuhan, China, 2002.

[2] B. Zeng, H. Duan, Y. Bai, and W. Meng, "Forecasting the output of shale gas in China using an unbiased grey model and weakening buffer operator," Energy, vol. 151, pp. 238-249, 2018.

[3] Z.-X. Wang and P. Hao, "An improved grey multivariable model for predicting industrial energy consumption in China," Applied Mathematical Modelling, vol. 40, no. 11-12, pp. 5745-5758, 2016.

[4] L. Kong and X. Ma, "Comparison study on the nonlinear parameter optimization of nonlinear grey Bernoulli model (NGBM $(1,1))$ between intelligent optimizers," Grey Systems: Theory and Application, vol. 8, no. 2, pp. 210-226, 2018.

[5] H. Duan and X. Luo, "A novel multivariable grey prediction model and its application in forecasting coal consumption," ISA Transactions, vol. 112, 2021.

[6] X. Xiao, J. Yang, S. Mao, and J. Wen, “An improved seasonal rolling grey forecasting model using a cycle truncation accumulated generating operation for traffic flow," Applied Mathematical Modelling, vol. 51, pp. 386-404, 2017.
[7] H. Duan and X. Xiao, "A multimode dynamic short-term traffic flow grey prediction model of high-dimension tensors," Complexity, vol. 2019, pp. 1-18, Article ID 9162163, 2019.

[8] H. Duan, X. Xiao, J. Long, and Y. Liu, “Tensor alternating least squares grey model and its application to short-term traffic flows," Applied Soft Computing, vol. 89, Article ID 106145, 2020.

[9] X. Xiao and H. Duan, "A new grey model for traffic flow mechanics," Engineering Applications of Artificial Intelligence, vol. 88, Article ID 103350, 2020.

[10] C. Yan, L. F. Wu, L. Y. Liu et al., "Fractional Hausdorff grey model and its properties," Chaos, Solitons and Fractals, vol. 138, Article ID 109915, 2020.

[11] Z.-X. Wang, Y.-F. Zhao, and L.-Y. He, "Forecasting the monthly iron ore import of China using a model combining empirical mode decomposition, non-linear autoregressive neural network, and autoregressive integrated moving average," Applied Soft Computing, vol. 94, Article ID 106475, 2020.

[12] Y. Bai, Y. Li, B. Zeng, C. Li, and J. Zhang, "Hourly PM2.5 concentration forecast using stacked autoencoder model with emphasis on seasonality," Journal of Cleaner Production, vol. 224, pp. 739-750, 2019.

[13] B. Zeng, H. Li, and X. Ma, "A novel multi-variable grey forecasting model and its application in forecasting the grain production in China," Computers \& Industrial Engineering, vol. 150, Article ID 106915, 2020.

[14] B. Zeng, X. Ma, and M. Zhou, "A new-structure grey Verhulst model for China's tight gas production forecasting," Applied Soft Computing, vol. 96, Article ID 106600, 2020.

[15] L. Liu and L. Wu, "Forecasting the renewable energy consumption of the European countries by an adjacent nonhomogeneous grey model," Applied Mathematical Modelling, vol. 89, pp. 1932-1948, 2021.

[16] Q. Xiao, M. Shan, M. Gao, X. Xiao, and M. Goh, "Parameter optimization for nonlinear grey Bernoulli model on biomass energy consumption prediction," Applied Soft Computing, vol. 95, Article ID 106538, 2020.

[17] W. Wu, X. Ma, Y. Zhang, W. Li, and Y. Wang, "A novel conformable fractional non-homogeneous grey model for forecasting carbon dioxide emissions of BRICS countries," Science of The Total Environment, vol. 707, Article ID 135447, 2020.

[18] G. M. Duman, E. Kongar, and S. M. Gupta, "Estimation of electronic waste using optimized multivariate grey models," Waste Management, vol. 95, pp. 241-249, 2019.

[19] N.-M. Xie, S.-F. Liu, Y.-J. Yang, and C.-Q. Yuan, "On novel grey forecasting model based on non-homogeneous index sequence," Applied Mathematical Modelling, vol. 37, no. 7, pp. 5059-5068, 2013.

[20] N. M. Xie and S. F. Liu, "Research on the non-homogenous discrete grey model and its parameter's properties," Journal of Systems Engineering and Electronics, vol. 30, no. 5, pp. 863867, 2008.

[21] Q. Xiao, M. Shan, M. Gao, X. Xiao, and H. Guo, "Evaluation of the coordination between China's technology and t," and Economic Development of Economy, vol. 27, no. 1, pp. 24-44, 2020.

[22] H. Duan, D. Wang, X. Pang, Y. Liu, and S. Zeng, "A novel forecasting atpproach based ta case report," Journal of Cleaner Production, vol. 260, Article ID 120929, 2020.

[23] S. Mao, Y. Kang, Y. Zhang, X. Xiao, and H. Zhu, "Fractional grey model based on non-singular exponential kernel and its application in the prediction of electronic waste precious metal content," ISA Transactions, vol. 107, pp. 12-26, 2020. 
[24] S. Mao, M. Zhu, X. Wang, and X. Xiao, "Grey-Lotka-Volterra model for the competition and cooperation between thirdparty online payment systems and online banking in China," Applied Soft Computing, vol. 95, Article ID 106501, 2020.

[25] Z. X. Wang, D. D. Li, and H. H. Zheng, "Model comparison of $\operatorname{GM}(1,1)$ and $\operatorname{DGM}(1,1)$ based on Monte-Carlo simulation," Physica A: Statistical Mechanics and Its Applications, vol. 542, Article ID 123341, 2019.

[26] X. Ma, W. Q. Wu, and Y. Y. Zhang, "Improved $\operatorname{GM}(1,1)$ model based on Simpson formula and its applications," Journal of Grey System, vol. 31, no. 4, pp. 33-46, 2019.

[27] Z.-q. Jia, Z.-f. Zhou, H.-j. Zhang, B. Li, and Y.-x. Zhang, "Forecast of coal consumption in Gansu Province based on Grey-Markov chain model," Energy, vol. 199, Article ID 117444, 2020.

[28] L. Wu, X. Gao, Y. Xiao, Y. Yang, and X. Chen, "Using a novel multi-variable grey model to forecast the electricity consumption of Shandong Province in China," Energy, vol. 157, pp. 327-335, 2018.

[29] Q. Wang, S. Li, and R. Li, "Will Trump's coal revival plan work? - comparison of results based on the optimal combined forecasting technique and an extended IPAT forecasting technique," Energy, vol. 169, pp. 762-775, 2019.

[30] H. Duan, G. R. Lei, and K. Shao, "Forecasting crude oil consumption in China using a grey prediction model with an optimal fractional-order accumulating operator," Complexity, vol. 201812 pages, 2018.

[31] X. Ma, X. Mei, W. Wu, X. Wu, and B. Zeng, "A novel fractional time delayed grey model with Grey Wolf Optimizer and its applications in forecasting the natural gas and coal consumption in Chongqing China," Energy, vol. 178, no. 1, pp. 487-507, 2019.

[32] Y. Bai, J. Xie, C. Liu, Y. Tao, B. Zeng, and C. Li, "Regression modeling for enterprise electricity consumption: a comparison of recurrent neural network and its variants," International Journal of Electrical Power \& Energy Systems, vol. 126, Article ID 106612, 2021.

[33] Z.-X. Wang, Z.-W. Wang, and Q. Li, "Forecasting the industrial solar energy consumption using a novel seasonal $\operatorname{GM}(1,1)$ model with dynamic seasonal adjustment factors," Energy, vol. 200, Article ID 117460, 2020.

[34] X. Luo, H. Duan, and L. He, "A novel Riccati equation grey model and its application in forecasting clean energy," Energy, vol. 205, Article ID 118085, 2020.

[35] P.-F. Pai and W.-C. Hong, "Support vector machines with simulated annealing algorithms in electricity load forecasting," Energy Conversion and Management, vol. 46, no. 17, pp. 2669-2688, 2005.

[36] B. Y. Guo, F. M. Zhang, and J. Li, "Grey landslide prediction model based on improved simulated annealing," Prospecting Science and Technology, vol. 8, no. 4, pp. 10-13, 2006. 